\title{
MEDIDAS DE DIVERSIDADE FUNCIONAL: UMA ESTRATÉGIA PARA A CONSERVAÇÃO DA BIODIVERSIDADE
}

\author{
MEASURES OF FUNCTIONAL DIVERSITY: A STRATEGY FOR BIODIVERSITY \\ CONSERVATION
}

\author{
MEDIDAS DE DIVERSIDAD FUNCIONAL: UNA ESTRATEGIA PARA LA \\ PRESERVACIÓN DE LA BIODIVERSIDAD
}

Gabriela Marques Peixoto ${ }^{1}$

\begin{abstract}
Resumo
Compreender os mecanismos e processos referentes aos padrões de distribuições das comunidades, além da relação entre diversidade biológica e ecossistemas, é um desafio para biólogos e ecólogos. Entretanto, diante das constantes ameaças antrópicas, medidas mitigadoras para conservação da biodiversidade se tornaram um ponto norteador em trabalhos sobre comunidades biológicas. A medida em que as espécies são perdidas, o funcionamento do ecossistema é afetado; logo, uma alta biodiversidade pode ser crucial para a estruturação de diferentes ecossistemas. Desta forma, o estudo apresenta uma contextualizaçao crítica sobre temas ligados à biodiversisdade e seus componentes. Investiga-se, na pesquisa, novas metodologias para mensurar a funcionalidade e aplicabilidade dos ecossistemas em um contexto de sustentabilidade ambiental.
\end{abstract}

Palavras-chave: Dversidade de espécies. Ecossistemas. Funcionalidade ambiental.

\begin{abstract}
Understanding the mechanisms and processes related to community distribution patterns, in addition to the relationship between biological diversity and ecosystems has been a challenge for biologists and environmentalists. However, regarding the constant human threats, mitigating measures for biodiversity conservation have become a guiding point in work with biological communities. As species are lost, the functioning of the ecosystem is affected, which leads us to think that high biodiversity can be crucial for structuring in different ecosystems. Thus, the study presents a critical context about issues related to biodiversity and its components. It is investigated, in the research, new methodologies to measure the functionality of ecosystems and their applicabilities in a context of environmental sustainability.
\end{abstract}

Keywords: Species diversity. Ecosystems. Environmental functionality.

\section{Resumen}

Comprender los mecanismos y procesos relativos a los modelos de distribución de las comunidades, más allá de la relación entre diversidad biológica y ecosistemas, es un reto para biólogos y ecólogos. Sin embargo, frente a las constantes amenazas antrópicas, medidas de mitigación para la conservación de la biodiversidad se han hecho referencia para trabajos sobre comunidades biológicas. En la medida que las especies se pierden, el funcionamiento del ecosistema se ve afectado; de manera que una alta biodiversidad puede ser crucial para la estructuración de diferentes ecosistemas. Así, este estudio presenta una revisión crítica de temas vinculados a la biodiversidad y sus componentes. Se busca, en esta investigación, definir metodologías para medir la funcionalidad y la aplicabilidad de los ecosistemas en un contexto de sostenibilidad ambiental.

Palabras-clave: Diversidad de especies. Ecosistemas. Funcionalidad ambiental.

\section{Introdução}

1 Doutora em Biologia (Ecologia) pelo Instituto Nacional de $\quad$ Pesquisa da Amazônia. $\quad$ E-mail:
gabriela.marquespd@gmail.com.


Compreender as relações existentes entre diversidade biológica e os elementos estruturadores dos ecossistemas é um desafio para biólogos e ambientalistas (ANGELINI, 1999; DALY \& FARLEY, 2004; DÍAZ \& CABIDO, 2001; LAVOREL \& GARNIER, 2002). O tema é um ponto norteador para a elaboração de medidas mitigadoras para a conservação de espécies. No entanto, apesar de toda gama de estudos referenciando o termo diversidade biológica, nenhum consenso quanto a sua definição foi estabelecido - o que gera inúmeras interpretações (HURLBERT, 1971; MARTINS; SANTOS, 1999; RICOTTA, 2005). Grande parte das definições existentes na literatura, e que são amplamente aceita por pesquisadores, ambientalistas e leigos, faz referência aos três grandes componentes da diversidade: genomas, espécies e ecossistemas (FITZHUGH, 2013). No entanto, outra dimensão adicional pode ser sugerida, desta vez interligando composição, estrutura e função (NOSS, 1990). Partindo desta linha de interpretação, Ricklefs (2010) propôs uma definição que incorpora todas as dimensões e sugere que a diversidade de espécies se refere à variação entre organismos e os sistemas ecológicos em todos os níveis. Isso incluiria a variação genética de populações, aspectos morfológicos e funcionais das espécies, além de incluir os processos ecossistêmicos que estruturam os biomas.

De modo limitado, o termo diversidade biológico se configura como um sinônimo para riqueza de espécies (MAGURRAN, 2004). Para isso, são usados artifícios, como curva de coletores e estimadores de riqueza. No entanto, estas métricas geram pesos iguais às espécies raras e abundantes, tornando-as pouco informativas. A primeira tentativa de expressar matematicamente a diversidade taxonômica foi proposta por Fischer et al. (1943), utilizando dados sobre borboletas e outros insetos. Foi proposto que a distribuição do número de indivíduos da espécie menos abundante até a espécie mais abundante poderia ser descrita por uma série logarítmica, o conhecido parâmetro alfa (MARTINS; SANTOS, 1999). Por sua vez, Simpson (1949) afirmou que o índice de diversidade $\alpha$ de Fisher seria uma medida do grau de concentração dos indivíduos em um dado grupo; deste modo, propôs-se um índice de concentração que poderia ser usado para comparar diferentes comunidades de modo independente do modelo de distribuição de abundância, que não fosse influenciado pelo tamanho amostral. Este índice indica a probabilidade de dois indivíduos retirados ao acaso da comunidade pertencerem a espécies diferentes, e se tornou bastante difundido na literatura (MELO, 2008). Outro índice de diversidade, que foi intensamente empregado nas pesquisas ecológicas, foi proposto por Shannon e Weaver (1949). Esse índice objetivava dar mais peso para espécies raras e seu cálculo era proveniente da Teoria da Informação. 
Por volta dos anos 70 , praticamente todos os estudos eram realizados por meio dos índices de Shannon ou Simpson (MAGURRAN, 2004); no entanto, diferentes combinações de riqueza de espécies e equitabilidade, em comunidades distintas, podem gerar exatamente o mesmo valor de diversidade (HURLBERT, 1971; MELO, 2008; MENDES et al., 2008), o que torna esses índices taxonômicos pouco informativos. Além disto, tais métricas taxonômicas não são capazes de esclarecer ou fornecer pistas sobre os diferentes processos que atuam nos ecossistemas - que levam à diversidade dentro das comunidades (DÍAZ; CABIDO, 2001; PETCHEY et al., 2004; CIANCIARUSO et al., 2009). Sendo assim, ao abordar os diferentes temas relacionados aos aspectos da biodiversidade e funcionalidade dos ecossistemas, este artigo se propõe a apresentar uma breve contextualização crítica sobre o histórico e avanços das pesquisas em diversidade funcional, elencando diferentes metodologias existentes na área, principalmente em uma perspectiva de conservação e manutenção dos ecossistemas.

\section{Material e método}

Para os objetivos propostos, neste estudo, foram realizadas pesquisas bibliográficas em capítulo de livros e artigos científicos, por meio de bases de dados como o portal periódicos CAPES e Google acadêmico. Como descritores para nossa pesquisa, utilizamos os termos "Functional diversity metrics" e "Functional diversity indices". Toda a contextualização teórica se baseia na perspectiva de futuras pesquisas que aliem a funcionalidade dos ecossistemas e a sustentabilidade ambiental.

\subsection{A funcionalidade dos ecossistemas}

Apesar das inúmeras definições existentes, podemos considerar o conceito de espécies como um conjunto de indivíduos que possuem características fenotípicas e comportamentais que determinam quando e onde elas podem existir, além das interações com indivíduos de outras espécies (MC GILL et al., 2006). À medida que se perdem espécies, o funcionamento dos ecossistemas é afetado. Isso indica que uma alta biodiversidade seria crucial para a estruturação dos diversos ecossistemas e sua supressão; consequentemente, essas perdas são um prejuízo ao funcionamento dos mesmos (PETCHEY; GASTON, 2002; CADOTTE et al., 2011).

Ao tratarmos de funcionalidade, traçamos um limiar entre espécie e ambiente. Sabe-se que as espécies são distintas quanto aos seus requisitos ambientais e interações com os 
ecossistemas. Em outras palavras, as espécies interagem por meios como predação, dispersão de sementes, fertilização de solos, decomposição de matéria orgânica, entre outros. Todos estes mecanismos são capazes de manter a funcionalidade de um ecossistema. Noss (1990, p. 357) distinguiu, explicitamente, aspectos funcionais a partir de aspectos estruturais da biodiversidade, ao traçar um limiar entre estrutura e o que significava a abordagem de função dentro de uma comunidade:

\footnotetext{
Estrutura é a organização física ou padrão de um sistema, da complexidade de um habitat como medida dentro das comunidades, padrões em manchas e outros elementos. Função envolve processos ecológicos e evolutivos, incluindo o fluxo gênico, perturbação e ciclagem dos nutrientes.
}

A prática de agrupamento das espécies, através dos seus aspectos funcionais, não é algo novo dentro da ecologia de comunidades (GLASSER et al., 1983; POLIS; STRONG, 1996, TILMAN, 2001). No entanto, novas formas matemáticas de mensurar tais características funcionais vêm sendo propostas ao longo dos últimos anos (PETCHEY; GASTON, 2002). Medir a diversidade funcional denota medir os atributos funcionais (DÍAZ; CABIDO, 2001; TILMAN, 2001; HOOPER et al., 2005; CIANCIARUSO et al., 2009) e avaliar as associações das espécies, suas atividades e interações que garantam a manutenção e existência dos processos dentro dos ecossistemas (HOOPER et al., 2005). Estes atributos funcionais podem ser qualitativos ou quantitativos e abrangem aspectos morfológicos, bioquímicos, comportamentais ou fenológicos das espécies (CADOTTE et al., 2011). Já que cada espécie apresenta um conjunto distinto de características uma das outras das outras, essas espécies apresentam também níveis de função dentro de uma comunidade biológica. Em teoria, as diferenças nas características dos indivíduos determinam como os mesmos vão utilizar os recursos. Assim, o aumento da eficiência na utilização de recursos pelos indivíduos em ambientes heterogêneos, por sua vez, acarreta aumento da função dentro do ecossistema em que estão inseridos (DÍAZ; CABIDO, 2001).

As variações das características, ou traits dos indíviduos podem ser alteradas por fatores genéticos e ambientais, o que pode afetar diretamente a saúde dos organismos, além de influenciar no sucesso de forrageamento, habilidade de fuga, aspectos reprodutivos, entre outros (DOBSON; MICHENER, 1995; DÍAZ et al., 2013). Sendo assim, Steele (1997) definiu diversidade funcional como a variação das diferentes respostas às mudanças bióticas frente às mudanças ambientais, sugerindo um alto grau de conexão entre as respostas ocasionadas por mudanças ambientais e interações com os processos ecológicos. Estas abordagens funcionais são bastante citadas entre os ecólogos (PETCHEY; GASTON, 2002), 
tornando-se fulcrais para o entendimento à respeito da estabilidade, aquisição de recursos, ciclagem de nutrientes e produtividade nas comunidades (MASON et al., 2003; PETCHEY; GASTON, 2006).

\subsection{Mensurando a diversidade funcional}

O número crescente de métodos descritos para o cálculo da diversidade funcional presentes na literatura (MASON et al., 2003; BOTTA-DUKÁT, 2005; RICOTTA, 2005; PETCHEY \& GASTON, 2006; DE BELLO et al., 2011), nos remete a acreditar que tais medidas têm se consolidando dentro da ecologia, abrindo um leque de possíbilidades e abordagens que capturem, da melhor forma, a funcionalidade das diferentes linhagens dentro das comunidades. Estas medidas são classificadas em categóricas e contínuas (CIANCIARUSO et al., 2009), sendo as categóricas baseadas no agrupamento dos indivíduos, de acordo com suas características funcionais, grupos funcionais ou riqueza de grupos funcionais (DÍAZ; CABIDO, 2001). Este agrupamento acontece de forma arbitrária o que limita sua predição, pois as inclusões dos indivíduos em seus determinados grupos; por vezes, parte-se do pressuposto de que todos os membros do grupo possuem características funcionais semelhantes (redundantes), e que membros de outros grupos são tidos como completamente diferentes - o que pode gerar resultados equivocados (PETCHEY; GASTON, 2006). Medidas contínuas, por sua vez, vêm trazendo novas perspectivas para mensurar a funcionalidade dos indivíduos, através de artefatos matemáticos capazes de prever, da melhor forma, as riquezas funcionais locais e regionais (LALIBERTÉ et al., 2014).

A primeira tentativa de quantificar a diversidade funcional, dento do escopo matemático, ficou conhecida pela sigla $F A D$ ou functional atribute diversity, elaborada por WALKER et al. (1999). Logo em seguida, outras métricas surgiram, como a entropia quadrática de Rao (RAO, 1982; BOTTA-DUKÁT, 2005), ou a Functional diversity ou simplesmente $F D$. A FD é considerada hoje uma medida relativamente simples que consiste na construção de uma matriz utilizando dados das espécies e seus atributos funcionais (ver PETCHEY; GASTON, 2002), e se tornou bastante difundida entre as pesquisas na última década.

Com o passar dos anos, outras medidas foram propostas, como a riqueza funcional (FRic), ou divergência funcional (FDiv) (VILLÉGER et al., 2008), além das medidas que incorporem os componentes espaciais, sejam padrões locais, ou regionais, (ANDERSON et al., 2006). Recentemente, um índice proposto por Villéger et al. (2008), vem ganhando 
destaque em diversas pesquisas ecológicas, conhecido por dissimilaridade funcional ou simplesmente FDIS. Esse índice é considerado como a distância média ponderada em um espaço multidimensional dos atributos de cada espécie para o centroide de todas as espécies de um dendograma funcional, onde os pesos correspondem à relação de abundâncias. O FDIS é considerado como uma alternativa para os índices de diversidade funcional, por permitir comparações entre a diversidade funcional de distintas localidades, semelhante à diversidade taxonômica $\beta$ (LALIBERTÉ; LEGENDRE, 2010; FRAGA et al., 2018).

Ainda mais recentemente, novas abordagens matemáticas capazes de incluir, não apenas as variações entre espécies, mas as diferenças entre os traços funcionais dos indivíduos de uma mesma população, começaram a surgir (DE BELLO et al., 2010). Esta pespectiva matemática parte do pressuposto de que muitos traços funcionais, responsáveis por influênciar diretamente o funcionamento dos ecossistemas, podem variar tanto inter quanto intraespecificamente (ALBERT et al., 2011), além de possuírem forte influência sobre os padrões de concorrência dos indivíduos, ciclagem de nutrientes (MADRITCH; HUNTER, 2003), resistência a perturbações (REUSCH et al., 2005), e produtividade dos ecossistemas (NORBERG et al., 2001). Com base nesta ideia, Cianciaruso et al., (2009) propôs uma nova abordagem matemática, conhecida pela sigla $i F D$, e assim foi capaz de incluir tal variação intraespecífica entre os atributos funcionais de uma mesma espécie, ampliando, desta forma, os métodos já existentes e consolidados. Para uma melhor comparação dos diferentes métodos citados na literatura atual, as vantagens em seu uso e suas limitações, ver Tabela 1.

Comumente, a escolha por diferentes medidas de diversidade funcional é dependente das perguntas e objetivos dos estudos, sejam eles apenas identificar a influência da biodiversidade sobre o funcionamento dos ecossistemas, ou mesmo elucidar os processos que regem os padrões de biodiversidade locais e regionais, as conhecidas regras de montagem das comunidades (SCHLEUTER et al., 2010; SOBRAL; CIANCIARUSO, 2012). Na perspectiva de conservação, estudos que avaliam o efeito de extinção de espécies na diversidade funcional, revelam que as comunidades geralmente contêm redundância limitada (PETCHEY; GASTON, 2002); dessa forma, infere-se que processos aleatórios de extinções afetariam a diversidade funcional. Além disso, comunidades com alta diversidade funcional são mais resistentes às invasões por espécies exóticas e os traços funcionais das espécies podem influenciar o sucesso desta invasão (SYMSTAD, 2000). Deste modo, estratégias eficazes para a conservação e restauração da biodiversidade são aquelas que priorizam a função do ecossistema ou estabilidade dos ambientes naturais (CADOTTE et al., 2011). Essas 
estratégias, no entanto, são mais eficazes quando interligadas aos aspectos da história natural e filogenéticos das espécies em áreas protegidas (BROOKS et al., 2006; LEE; JETZ, 2008).

De fato, o uso de múltiplas medidas de diversidade - em diferentes escalas de observação — podem ajudar a compreender a importância relativa dos processos históricos e ecológicos na formação das comunidades (FRAGA et al., 2018). Entretanto, para as características funcionais, alguns traços apresentam forte sinal filogenético, o que nos permite inferir que a história evolutiva se relaciona com a evolução dos traços. Por isso, a escolha dos traços a serem mensurados precisa ser cuidadosamente avaliada, integrando uma quantidade e qualidade significativa de características que garantam a captura dos aspectos que afetam diretamente nos processos ecossistêmicos.

Apesar da vasta literatura sobre a diversidade funcional, suas implicações e metodologias (CHALCRAFT; RESETARITS, 2003; RESETARITS; CHALCRAFT, 2007; JAIN et al., 2013; BERRIOZABAL-ISLAS et al., 2017.), diversos táxons permanecem sem referencial teórico sobre o tema, o que se configura como um leque de oportunidades para as pesquisas ecológicas; contudo, isso dificulta ainda mais as escolhas dos atributos a serem escolhidos. De fato, diferentes características funcionais têm níveis distintos de redundância dentro das comunidades biológicas. Somente traços relacionados com a função de interesse devem ser contidos nas métricas, já que inclusões excessivas de características neutras ou positivamente correlacionados levam a uma convergência artificial, entre a diversidade funcional e outros índices taxonômicos, enquanto traços correlacionados negativamente irão inflar os valores de diversidade funcional (PAVOINE et al., 2011), além de gerar resultados equivocados.

Saber usar os artifícios matemáticos para medir aspectos ambientais requer cuidado e planejamento, a fim de evitar resultados distorcidos e uma magnitude de informações perdidas. Partindo desta ideia, quando tentamos calcular a diversidade funcional, a primeira etapa para montar um dendograma funcional das espécies começa pelas escolhas dos traços. Necessita-se, assim, que cada característica seja inserida levando em consideração a função que ela desempenha no ecossistema, sejam referentes à aquisição, uso e disponibilidade de recursos, ou as que afetam a ocorrência e expansão dos distúrbios (TILMAN, 2001). O segundo passo é a escolha da melhor métrica e isso depende das perguntas que se pretende responder e a escala das amostragens, sejam locais ou regionais. A adequação da escala espacial de ocorrência das espécies, desempenha papel crucial nos processos que afetam a composição e aspectos funcionais das espécies, podendo alterar e, até mesmo, mascarar resultados (SCHNEIDER, 2001). A compreenção dos processos envolvidos na distribuição da 
biodiversidade em diferentes escalas é de suma importância para a ecologia e biogeografia, já que essa compreensão pode afetar a visão dos resultados (GASTON; BLACKBURN, 2003), e perfaz decisiva para a conservação.

Tabela 1. Comparação dos principais índices de diversidade funcional citados na literatura atual e as vantagens e desvantagens de uso de cada método.

\begin{tabular}{|c|c|c|c|}
\hline $\begin{array}{c}\text { Índice } \\
\text { Abreviação/Nome }\end{array}$ & Vantagens & Desvantagens & $\begin{array}{l}\text { Artigo de } \\
\text { referência }\end{array}$ \\
\hline $\begin{array}{l}\text { FD/Dendrograma } \\
\text { funcional }\end{array}$ & $\begin{array}{c}\text { Uso de múltiplos traços } \\
\text { e variáveis categóricas; } \\
\text { não inclui a abundância } \\
\text { de espécies. }\end{array}$ & $\begin{array}{l}\text { Muito dependente da escolha } \\
\text { da distância e do método de } \\
\text { agrupamento a ser utilizado, } \\
\text { o que pode afetar os valores } \\
\text { obtidos. É dependente da } \\
\text { riqueza de espécies. }\end{array}$ & $\begin{array}{c}\text { Petchey \& Gaston } \\
\text { (2002). }\end{array}$ \\
\hline $\begin{array}{c}\mathbf{F D}_{\mathbf{Q}} / \text { Entropia } \\
\text { quadrática de Rao's }\end{array}$ & $\begin{array}{c}\text { Usa variáveis } \\
\text { categóricas; não é } \\
\text { dependente da riqueza } \\
\text { de espécies. }\end{array}$ & $\begin{array}{c}\text { É dependente da abundância } \\
\text { das espécies. }\end{array}$ & $\begin{array}{c}\text { Rao (1982), } \\
\text { adaptado por } \\
\text { Botta-Dukát } \\
(2005) .\end{array}$ \\
\hline $\begin{array}{c}\text { FEve / } \\
\text { Equitabilidade } \\
\text { functional }\end{array}$ & $\begin{array}{c}\text { Usa variáveis } \\
\text { categóricas; não é } \\
\text { dependente da riqueza } \\
\text { de espécies. }\end{array}$ & $\begin{array}{c}\text { É dependente da abundância } \\
\text { das espécies. }\end{array}$ & $\begin{array}{l}\text { Villeger et al. } \\
\text { (2008). }\end{array}$ \\
\hline $\begin{array}{l}\text { FRic/ Riqueza } \\
\text { funcional }\end{array}$ & $\begin{array}{l}\text { Usa variáveis } \\
\text { categóricas. }\end{array}$ & $\begin{array}{l}\text { Não usa múltiplos traços; } \\
\text { tem forte relação positiva } \\
\text { com a riqueza de espécies. }\end{array}$ & $\begin{array}{l}\text { Mason et al. } \\
\quad \text { (2005). }\end{array}$ \\
\hline $\begin{array}{l}\text { FDiv/ Divergência } \\
\text { funcional }\end{array}$ & $\begin{array}{c}\text { Índice } \\
\text { multidimensional } \\
\text { baseado na dispersão de } \\
\text { múltiplos traços; não é } \\
\text { dependente da riqueza } \\
\text { de espécies. }\end{array}$ & $\begin{array}{c}\text { É dependente da abundância } \\
\text { das espécies. }\end{array}$ & $\begin{array}{l}\text { Mason et al. } \\
\quad \text { (2003). }\end{array}$ \\
\hline $\begin{array}{l}\text { FDIS/ Dispersão } \\
\text { funcional }\end{array}$ & $\begin{array}{c}\text { Índice } \\
\text { multidimensional } \\
\text { baseado na dispersão de } \\
\text { múltiplos traços; Não é } \\
\text { dependente da riqueza } \\
\text { de espécies. }\end{array}$ & $\begin{array}{c}\text { É dependente da abundância } \\
\text { das espécies. }\end{array}$ & $\begin{array}{c}\text { Laliberté \& } \\
\text { Legendre (2010). }\end{array}$ \\
\hline
\end{tabular}




\section{Considerações finais}

Diante do exposto, a diversidade funcional é uma alternativa plausível para predizer os padrões de ocorrência das espécies e, consequentemente, as regras de estruturação das comunidades biológicas - já que a variação dos processos ecológicos tem forte influência do número e tipos de espécies existentes em um determinado ambiente. Entretanto, esta abordagem apresenta ressalvas quanto a escolha dos traços funcionais, das métrica que serão aplicadas, ou mesmo o mapeamento da atuação de possíveis filtros ambientais, que por vezes, são capazes de mascarar a verdadeira relação entre os índices. Deste modo, um desenho amostral eficiente, alinhado a diferentes escalas, é a forma mais eficiente de se trabalhar com a mensuração da funcionalidade dos ecossistemas, prever respostas e para a aplicabilidade no contexto de conservação da biodiversidade.

\section{Referências}

ALBERT, Cécile H. et al. On the importance of intraspecific variability for the quantification functional diversity. Nordic Society Oikos, [s. 1], v. 121, p. 116-126, 2011. DOI: https://onlinelibrary.wiley.com/doi/epdf/10.1111/j.1600-0706.2011.19672.x.

ANDERSON, Marti J.; ELLINGSEN, Kari E.; MCARDLE, Brian. H. Multivariate dispersion as a measure of beta diversity. Ecology Letters, França, v. 9, p. 683-693, 2006. DOI: https://onlinelibrary.wiley.com/doi/epdf/10.1111/j.1461-0248.2006.00926.x

ANGELINI, Ronaldo. Ecossistemas e modelagem. In: POMPÊO, Marcelo Luiz Martins. Perspectivas da Limnologia no Brasil. São Luís: Gráfica e Editora União, 1999. 198 p.

BERRIOZABAL-ISLAS, Christian et al. Effects of habitat disturbance on lizard functional diversity in a tropical dry forest of the Pacific Coast of Mexico. Tropical Conservation Science, [s. 1], v. 10, p. 1-11, 2017. DOI: https://doi.org/10.1177/1940082917704972.

BOTTA-DUKÁT, Zoltán. Rao's quadratic entropy as a measure of functional diversity based on multiple traits. Journal Vegetation Science, [s. 1], v. 16, n. 5, p. 533-540, 2005.

BROOKS, Thomas M. et al. Global biodiversity conservation priorities. Science, [s. 1], v. 313, n. 5783, p. 58-61, 2006. DOI: 10.1126/science.1127609.

CADOTTE, Marc W.; CARSCADDEN, Kelly; MIROTCHNICK, Nicholas. Beyond species: functional diversity and the maintenance of ecological processes and services. Journal of Applied Ecology, [s. 1], v. 48, n. 5, p. 1079-1087, 2011

CHALCRAFT, David R.; RESETARITS, JR, William J. Mapping functional similarity of predators on the basis of trait similarities. The American Naturalist, [s. 1], v. 162, n. 4, p. 390-402, 2003. 
CIANCIARUSO, Marcus Vinicius et al. Including intra specific variability in functional diversity. Ecology, [s. 1], v. 90, n. 1, p. 81-89, 2009.

DALY, Herman E.; FARLEY, Joshua. Ecological Economics: principles and applications. Washington DC: Island Press, 2004.

DE BELLO, Francesco et al. Towards an assessment of multiple ecosystem processes and services via functional traits. Biodiversity and Conservation, [s. 1], v. 19, n. 10, p. 28732893, 2010. DOI: https://doi.org/10.1007/s10531-010-9850-9.

DE BELLO, Francesco et al. Quantifying the relevance of intraspecific trait variability for functional diversity. Methods in Ecology and Evolution, [s. 1], v. 2, n. 2, p. 163-174, 2011. DOI: https://doi.org/10.1111/j.2041-210X.2010.00071.x.

DÍAZ, Sandra et al. Functional traits, the phylogeny of function, and ecosystem service vulnerability. Ecology and Evolution, [s. 1], v, 3, n. 9, p. 2958-2975, 2013.

DÍAZ, Sandra; CABIDO, Marcelo. Vive La différence: plant functional diversity matters to ecosystem processes. Trends in Ecology and Evolution, [s. 1], v. 16, n. 11, p. 646-655, 2001. DOI: https://doi.org/10.1016/S0169-5347(01)02283-2.

DOBSON, F. Stephen; MICHENER, Gail R. Maternal traits and reproduction in Richardson's ground squirrels. Ecology, Washington DC, v. 18, n. 73, p. 851-862, 1995. DOI: https://doi.org/10.2307/1939350.

FISHER, Ronald A.; CORBET, A. Steven; WILLIAMS, Carrington B. The relation between the number of species and the number of individuals in a random sample of an animal population. Journal Animal Ecology, [s. 1], v. 12, n. 1, p. 42-58, 1943. DOI:

https://www.jstor.org/stable/1411.

FITZHUGH, Kirk. Defining 'Species,' 'Biodiversity,' and 'Conservation' by Their Transitive Relation. In: The Species Problem: ongoing issues. Rijeka, Croatia: IntechOpen, 2013. 95$130 \mathrm{p}$.

FRAGA, RAFAEL et al. Different environmental gradients affect different measures of snake $\beta$-diversity in the Amazon rainforests. PeerJ. [s. 1], v. 6, p. 1-18, 2018. Disponível em: https://research-management.mq.edu.au/ws/portalfiles/portal/93946436/93061786.pdf. Acesso em: 27 maio 2019.

GASTON, Kevin J.; BLACKBURN, Tim M. Macroecology and conservation biology. In: Blackburn Tim M., Gaston Kevin J. Macroecology: Concepts and consequences. Oxford: Blackwell Science, 2003. 300 p.

GLASSER, Wolfgang G. et al. The chemistry of several novel bioconversion lignins. Journal of Agricultural and Food Chemistry, [s. 1], v. 31, n. 5, p. 921-930, 1983. DOI: https://pubs.acs.org/doi/pdf/10.1021/jf00119a001.

HOOPER, David U. et al. Effects of biodiversity on ecosystem functioning: a consensus of current knowledge. Ecological monographs, [s. 1], v. 75, n. 1, p. 3-35, 2005. DOI: https://esajournals.onlinelibrary.wiley.com/doi/abs/10.1890/04-0922. 
HURLBERT, Stuart H. The nonconcept of species diversity: a critique and alternative parameters. Ecology, v. 52, n. 4, p. 577-586, 1971. DOI:

https://esajournals.onlinelibrary.wiley.com/doi/abs/10.2307/1934145.

JAIN, Meha et al. The importance of rare species: a trait-based assessment of rare species contributions to functional diversity and possible ecosystem function in tall-grass prairies. Ecology and Evolution, [s. 1], v. 4, n. 1, p. 104-112, 2013. DOI:

https://onlinelibrary.wiley.com/doi/full/10.1002/ece3.915.

LALIBERTÉ, Etienne.; LEGENDRE, Pierre. A distance-based framework for measuring functional diversity from multiple traits. Ecology, [s. 1], v. 91, n. 1, p. 299-305, 2010. DOI: https://doi.org/10.1890/08-2244.1.

LALIBERTÉ, Etienne et al. Measuring functional diversity from multiple traits, and other tools for functional ecology. 2014. Disponível em:

https://mran.microsoft.com/snapshot/2014-11-17/web/packages/FD/FD.pdf. Acesso em: 27 maio 2019.

LAVOREL, Sandra; GARNIER, Eric. Predicting changes in community composition and ecosystem functioning from plant traits: revisiting the Holy Grail. Functional Ecology, [s. 1], v. 16, n. 5, p. 545-556, 2002.

LEE, Tien Ming; JETZ, Walter. Future battlegrounds for conservation under global change. Proc. R. Soc. London B, [s. 1], v. 275, p.1261-1270, 2008. DOI: https://royalsocietypublishing.org/doi/full/10.1098/rspb.2007.1732.

MADRITCH, Michael D.; HUNTER, Mark D. Intraspecific litter diversity and nitrogen deposition affect nutrient dynamics and soil respiration. Oecologia, [s. 1], v. 136, n. 1, p. 124128, 2003. Disponível em: https://link.springer.com/content/pdf/10.1007/s00442-003-12530.pdf. Acesso em: 28 mai. 2019.

MAGURRAN, Anne E. Measuring biological diversity. Oxford: Blackwell, 2004. 256 p.

MARTINS, Fernando Roberto; SANTOS, Flavio Antonio Maës dos. Técnicas usuais de estimativa da biodiversidade. Revista Holos, Campinas, p. 236-267. 1999. Disponível em: http://www.lerf.eco.br/img/publicacoes/2000_3011\%20Tecnicas\%20usuais\%20de\%20estimat iva\%20da\%20biodiversidade.pdf. Acesso em: 28 maio 2019.

MASON, Norman WH. et al. An index of functional diversity. Journal of Vegetation Science, [s. 1], v. 14, n. 4, p. 571-578, 2003. DOI: https://doi.org/10.1111/j.16541103.2003.tb02184.x

MASON, Norman WH. et al. Functional richness, functional evenness and functional divergence: the primary components of functional diversity. Oikos, [s. 1], v. 111, n. 1, p. 112118, 2005. DOI: https://doi.org/10.1111/j.0030-1299.2005.13886.x.

McGILL, Brian J. et al. Rebuilding community ecology from functional traits. Trends in Ecology \& Evolution, [s. 1], v. 21, n. 4, p. 178-185, 2006. DOI:

https://doi.org/10.1016/j.tree.2006.02.002. 
MELO, A. S. What do we win 'confounding' species richness and evenness in a diversity index? Biota Neotropica, [s. 1], v. 8, n. 1, p. 21-27, 2008.

MENDES, Renio S. et al. A unified index to measure ecological diversity and species rarity. Ecography, [s. 1], v. 31, n. 4, p. 450-456, 2008. DOI: https://doi.org/10.1111/j.09067590.2008.05469.x.

NORBERG, Jon et al. Phenotypic diversity and ecosystem functioning in changing environments: a theoretical framework. Proceedings of the National Academy of Sciences, [s. l], v. 98, n. 20, p. 11376-11381, 2001. DOI: https://doi.org/10.1073/pnas.171315998.

NOSS, Reed F. Indicators for monitoring biodiversity: a hierarchical approach. Conservation biology, [s. 1], v. 4, n. 4, p. 355-354, 1990. DOI: https://doi.org/10.1111/j.15231739.1990.tb00309.x.

PAVOINE, Sandrine; DUFOUR, Anne-Béatrice; CHESSEL, Daniel. From dissimilarities among species to dissimilarities among communities: a double principal coordinate analysis. Journal of Theoretical Biology, [s. 1], v. 228, n. 4, p. 523-537, 2004. DOI: https://doi.org/10.1016/j.jtbi.2004.02.014.

PETCHEY, Owen L.; GASTON, Kevin J. Functional diversity (FD), species richness and community composition. Ecology letters, [s. 1], v. 5, n. 3, p. 402-411, 2002.

PETCHEY, Owen L.; HECTOR, Andy; GASTON, Kevin J. How do different measures of functional diversity perform? Ecology, [s. 1], v. 85, n. 3, p. 847-857, 2004. DOI: https://doi.org/10.1890/03-0226.

PETCHEY, Owen L.; GASTON, Kevin J. Functional diversity: back to basics and looking forward. Ecology Letters, [s. 1], v. 9, n. 6, p. 741-758, 2006. DOI: https://doi.org/10.1111/j.1461-0248.2006.00924.x.

POLIS, Gary A.; STRONG, Donald R. Food web complexity and community dynamics. American Naturalist, [s. 1], v. 147, n. 5, p. 813-846, 1996. DOI: https://www.journals.uchicago.edu/doi/citedby/10.1086/285880.

RAO, C. R. Diversity and dissimilarity coefficients-a unified approach. Theoretical Population Biology, [s. 1], v. 21, n. 1, p. 24-43, 1982. DOI: https://doi.org/10.1016/00405809(82)90004-1.

RESETARITS, William J.; CHALCRAFT, David R. Functional diversity within a morphologically conservative genus of predators: implications for functional equivalence and redundancy in ecological communities. Functional Ecology, [s. 1], v. 21, n. 4, p. 793-804, 2007. DOI: https://doi.org/10.1111/j.1365-2435.2007.01282.x.

REUSCH, Thorsten B. H et al. Ecosystem recovery after climatic extremes enhanced by genotypic diversity. P. Natl. Acad. Sci, [s. 1], v. 102, n. 8, p. 2826-2831, 2005. DOI: https://doi.org/10.1073/pnas.0500008102.

RICKLEFS, Robert E. (2010) Evolutionary diversification, coevolution between populations and their antagonists, and the filling of niche space. Proceedings of the National Academy 
of Sciences, [s. 1], v. 107, n. 4, p. 1265-1272, 2010. DOI: https://doi.org/10.1073/pnas.0913626107.

RICOTTA, Carlo. 2005. Additive partitioning of Rao quadratic diversity: a hierarchical approach. Ecol. Model., [s. 1], v. 183, n. 4, p. 365-371, 2005. DOI:

https://doi.org/10.1016/j.ecolmodel.2004.08.020.

SCHLEUTER, D. et al. A user's guide to functional diversity indices. Ecological

Monographs, [s. 1], v. 80, n. 3, p. 469-484, 2010. DOI: https://doi.org/10.1890/08-2225.1.

SCHNEIDER, David C. The rise of the concept of scale in Ecology. Bioscience, [s. 1], v. 51, n. 7, p. 545-556, 2001. DOI: https://doi.org/10.1641/0006-

3568(2001)051[0545:TROTCO]2.0.CO;2.

SHANNON, C. E.; WEAVER, W. The mathematical theory of communication. Illinois: University of Illinois, 1949.

SIMPSON, Edward H. Measurement of diversity. Nature, [s. 1], v. 163, n. 4148, p. 688-688, 1949. DOI: https://doi.org/10.1038/163688a0.

SOBRAL, Fernando Landa; CIANCIARUSO, Marcus Vinicius. Estrutura filogenética e funcional de assembleias:(re) montando a Ecologia de Comunidades em diferentes escalas espaciais. Bioscience Journal, [s. 1], v. 28, n. 4, 18 jun. 2012. Disponível em:

http://www.seer.ufu.br/index.php/biosciencejournal/article/view/13491. Acesso em: 27 maio 2019.

TEELE, Claude M. A threat in the air: How stereotypes shape intellectual identity and performance. American psychologist, [s. 1], v. 52, n. 6, p. 613, 1997. DOI:

https://doi.org/10.1037/0003-066X.52.6.613.

SYMSTAD, Amy J. A test of the effects of functional group richness and composition on grassland invasibility. Ecology, [s. 1], v. 81, n. 1, p. 99-109, 2000. DOI:

https://doi.org/10.1890/0012-9658(2000)081[0099:ATOTEO]2.0.CO;2.

TILMAN, David. Functional diversity. 2001. Disponível em:

https://www.cedarcreek.umn.edu/sites/default/files/public/t1797.pdf. Acesso em: 27 maio 2019.

VILLÉGER, Sébastien; MASON, Norman WH; MOUILLOT, David. New multidimensional functional diversity indices for a multifaceted framework in functional ecology. Ecology, [s. l], v. 89, n. 8, p. 2290-2301, 2008. DOI: https://doi.org/10.1890/07-1206.1.

WALKER, Brian; KINZIG, Ann; LANGRIDGE, Jenny. Plant attribute diversity, resilience, and ecosystem function: the nature and significance of dominant and minor species.

Ecosystems, [s. 1], v. 2, n. 2, p. 95-113, 1999. Disponível em:

https://link.springer.com/content/pdf/10.1007/s100219900062.pdf. Acesso em: 27 maio 2019. 